\title{
Unilateral gynecomastia as an initial presentation of hyperthyroid Graves' disease
}

\section{Titipatima Sakulterdkiat ${ }^{1}$, Kessanee Romphothong(2), Waralee Chatchomchuan'2, Soontaree Nakasatien ${ }^{2}$, Sirinate Krittiyawong ${ }^{2}$, Yotsapon Thewjitcharoen $\mathbb{D}^{2}$ and Thep Himathongkam²}

'Pathobiology Department, Faculty of Science, Mahidol University, Bangkok, Thailand and 2Diabetes and Thyroid Center, Theptarin Hospital, Bangkok, Thailand
Correspondence should be addressed to $Y$ Thewjitcharoen Email

kamijoa@hotmail.com

\section{Summary}

Graves' disease is an autoimmune condition leading to the activation of and an increase in thyroid hormone secretion. Manifestations of hyperthyroidism in Graves' disease can vary among people. In this case, we report a 24-year-old Thai man with a rare presentation of unilateral gynecomastia along with symptoms of thyrotoxicosis. Physical examination revealed a $3 \mathrm{~cm}$ non-tender palpable glandular tissue beneath and around the left areola without nipple discharge and moderately diffuse thyroid enlargement with thyroid bruit. Thyroid function test showed a typical thyrotoxicosis state with elevated serum-free T4 and decreased serum TSH. His diagnosis of Graves' disease was confirmed biochemically with a highly elevated anti-TSH receptor antibody. Early treatment with anti-thyroid medication was given first, followed by Radioiodine treatment (RAl) for definitive treatment due to high level of anti-TSH receptor antibody, enlarged thyroid and severe thyrotoxicosis presentation at a young age, which might not resolve by anti-thyroid medication alone. The patient responded well to treatment and achieved complete resolution of unilateral gynecomastia with clinically and biochemically euthyroid within 3 months after treatment. No recurrent gynecomastia was found during the 2-year follow-up.

\section{Learning points:}

- Characteristic of gynecomastia in hyperthyroidism is usually presented with bilateral progressive gynecomastia; however, unilateral gynecomastia is occasionally found as a presentation of hyperthyroidism.

- Complete resolution of gynecomastia without recurrence can be achieved within a few months of treatment after thyrotoxicosis is resolved in patients with hyperthyroidism with the recent development of gynecomastia.

- RAI for definitive treatment is recommended in young adult patients expressing very high anti-TSH antibody level with severe thyrotoxicosis.

\section{Background}

Graves' disease (GD) is a result of autoimmune antibody stimulation of TSH receptor resulting in increased production and release of thyroid hormone and is the most common cause of hyperthyroidism. Presentation of hyperthyroidism varies between individuals, with patients displaying clinical symptoms of weight loss, fatigue, heat intolerance, tremor and palpitations as well as thyroidassociated ophthalmopathy. Symptoms of bilateral gynecomastia in association with GD and thyrotoxicosis can be found in various reported clinical cases as frequently as $40 \%$ (1). However, the presentation of unilateral gynecomastia in association with hyperthyroidism remains rare.

Gynecomastia in men results from an increased circulatory level of estrogen that subsequently stimulates inappropriate growth of breast tissue. The disproportion 
of estrogen and androgen caused by three mechanisms includes: an increase in estrogen levels, a decrease in androgen level and an increase in sex hormone-binding globulin level. The balance between free androgen and estrogen in patients with hyperthyroidism is affected by elevated serum levels of sex hormone-binding globulin; therefore, patients with hyperthyroidism can have an increased ratio of estrogen to androgen leading to the clinical presentation of gynecomastia.

The most common cause of unilateral gynecomastia is breast tumor formation (2); interestingly, we describe here a case of Thai male with the presentation of unilateral gynecomastia due to GD. An appropriate diagnosis was essential for this patient with atypical clinical symptom since recognizing the presentation of unilateral gynecomastia as presentation of hyperthyroidism could hasten definitive diagnosis and therapy for this reversible condition and avoid any unnecessary investigation for fear of malignancy.

\section{Case presentation}

A 24-year-old Thai man presented with non-painful enlargement of the left breast along with symptoms of palpitation, excessive sweating and weight loss of $3 \mathrm{~kg}$ over the duration of 1 month. His birth and childhood history were unremarkable. He reported the onset of puberty at 13 years. He had normal libido and a normal frequency of shaving. He revealed no alcohol or drug abuse history. He was not taking any medication. He denied a family history of gynecomastia or cancer. Vital signs revealed tachycardia with a heart rate of 115 b.p.m with otherwise normal ECG. Body temperature, blood pressure, respiratory rate and oxygen saturation were all within normal limits. His height was $173 \mathrm{~cm}$ and weighed $52.9 \mathrm{~kg}$ with calculated BMI of $17.68 \mathrm{~kg} / \mathrm{m}^{2}$. Physical examination (Figs 1 and $2 \mathrm{~A}$ ) revealed a $3 \mathrm{~cm}$ non-tender palpable glandular tissue with firm consistency beneath and around the left areola without nipple discharge. There was no palpable mass at left breast. No evidence of Graves' ophthalmopathy. Moderately diffuse thyroid enlargement with the presence of bruit was detected. His tanner staging is $\mathrm{V}$, and he had a normal general examination and normal-sized testes with no detection of testicular mass. The testicular ultrasound was not performed because the physical examination of both testes did not reveal a testicular mass. Testicular exam during 3-year follow-up period also showed no testicular mass.

\section{Investigation}

Appropriate biochemical investigation was critical for the diagnosis of unilateral gynecomastia in this patient. Lab investigations to find the cause of gynecomastia were sent as follows: (i) estrogen level for the evaluation of increased estrogen activity; (ii) testosterone level for the evaluation of conditions associated with decreased androgen and LH and (iii) thyroid functions. Based on clinical presentation and initial laboratory investigation provided in Table 1, unilateral gynecomastia as a presentation of GD was suspected from a typical thyrotoxicosis state with elevated serum-free T4 and decreased serum TSH. No inconspicuous mass was detected, and no history of cancer was suspected; therefore, mammogram imaging was not requested. His diagnosis of GD was confirmed biochemically with a highly elevated anti-TSH receptor antibody. Furthermore, elevated serum estradiol and total testosterone in addition to elevated thyroid function support gynecomastia as a presentation of hyperthyroidism in this case.

\section{Treatment}

Following initial treatment with $40 \mathrm{mg}$ methimazole using dose titration regimen for 2 weeks, the patient's laboratory findings were already showing significant improvement with a decrease in the level of free T4 to normal range from $>7.7$ to $1.4 \mathrm{ng} / \mathrm{dL}(100.02-18.02 \mathrm{pmol} / \mathrm{L})$, level of T3 was still slightly above the normal range but also showed a significant decrease from $>651$ to $182 \mathrm{ng} / \mathrm{dL}$ (10.00-2.81 $\mathrm{nmol} / \mathrm{L}$ ) while the level of estradiol decreased from $88.1 \mathrm{pg} /$ $\mathrm{mL}(323.45 \mathrm{pmol} / \mathrm{L})$ to normal range of $51.6 \mathrm{pg} / \mathrm{ml}(189.44$ $\mathrm{pmol} / \mathrm{L}$ ) and level of SHBG were within normal limits (Table 1). Clinical examination at follow-up appointments with the patient correlated with the laboratory findings,
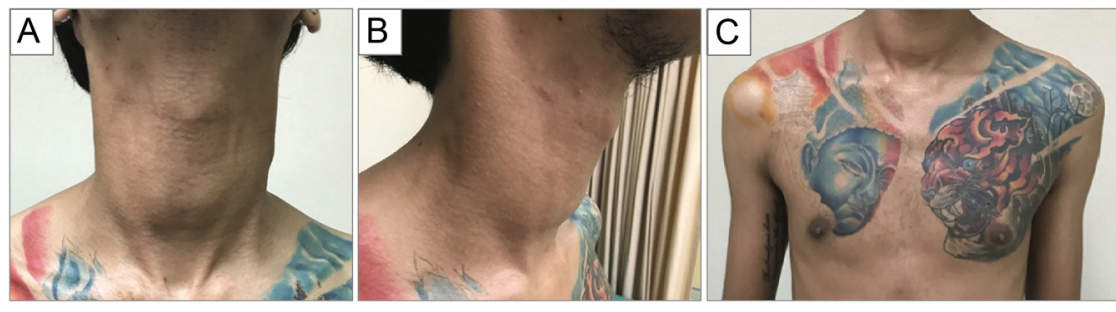

Figure 1

General appearance of hyperthyroid patient presented with (A) frontal and (B) side view of mild diffuse enlargement of thyroid and (C) manifestation of left unilateral gynecomastia.

https://edm.bioscientifica.com/ 

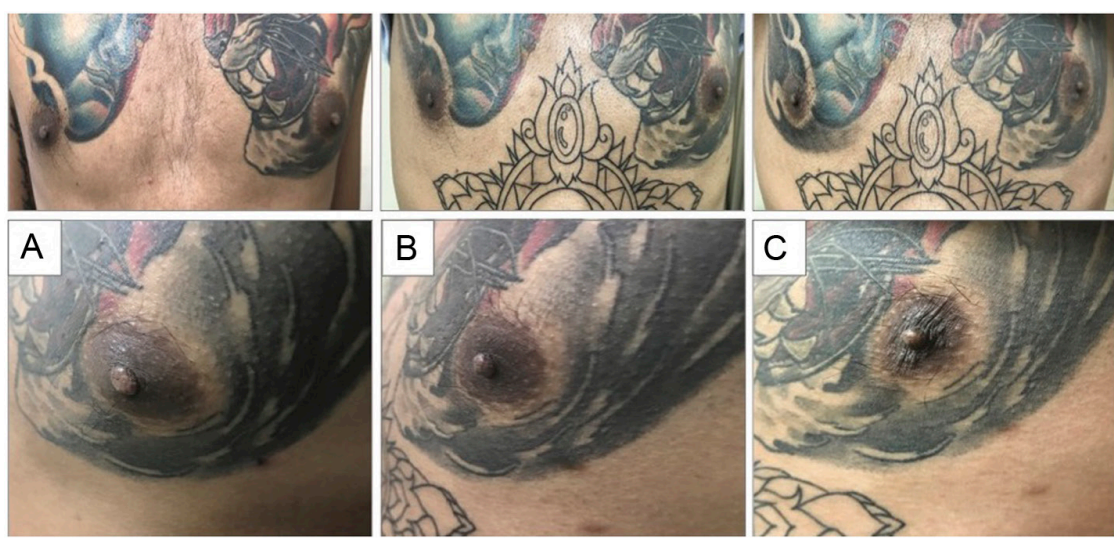

Figure 2

Clinical course following the treatment of unilateral gynecomastia in Graves' disease at (A) initial presentation and (B) after successive treatment with radioiodine (RAI) treatment at 2 weeks and (C) complete disappearance of gynecomastia after 3 months of treatment. showing improvement of gynecomastia symptoms at initial presentation (Fig. 2A) compared to 2 weeks after treatment (Fig. 2B). Then, $30 \mathrm{mCI}$ radioiodine was given as definitive treatment for GD in this patient since he had a high expression level of anti-TSH receptor antibody and displayed symptoms of thyrotoxicosis at a young age. RAI treatment continued for 2 months and rendered the patient to have a brief episode of hypothyroidism prompting treatment with levothyroxine until euthyroid was obtained and symptoms of gynecomastia were completely resolved (Fig. 2C).

\section{Outcome and follow-up}

Following treatment, the patient remained in euthyroid state for 2 years without recurrent gynecomastia. Thyroid hormone supplement was continued due to post-ablative hypothyroid, but symptoms of hyperthyroidism were completely resolved.

\section{Discussion}

Gynecomastia is defined as an enlargement of male breast tissue that can be due to either physiologic or pathologic condition. Physiologic gynecomastia can occur during infancy, puberty and advanced age. Any development of the male breast, especially during reproductive age, is often pathologic and underlying causes must be investigated (3). The mechanism of breast development in male is analogous to those of female as mammary cells are responsive to levels of estrogen and progesterone. An imbalance between estrogen and testosterone within the circulatory system or within the local breast tissue can cause pathologic gynecomastia (4). The mechanisms leading

Table 1 Laboratory investigation in a patient with unilateral gynecomastia as a symptom of hyperthyroidism Graves' disease and evolution of biochemical changes during the course of treatment.

\begin{tabular}{|c|c|c|c|c|c|c|}
\hline \multirow[b]{2}{*}{ Parameters } & \multirow[b]{2}{*}{ Normal values } & \multirow[b]{2}{*}{ Initial presentation } & \multicolumn{2}{|c|}{$\begin{array}{l}\text { After treatment with } \\
\text { methimazole }\end{array}$} & \multicolumn{2}{|c|}{$\begin{array}{l}\text { After treatment with } \\
\text { methimazole and RAI }\end{array}$} \\
\hline & & & 1 week & 3 weeks & 3 months & 6 months \\
\hline $\mathrm{TSH}(\mu \mathrm{IU} / \mathrm{mL})$ & $0.27-4.20$ & 0.01 & 5.48 & & $<0.01$ & 7.26 \\
\hline \multicolumn{7}{|l|}{ FT4 } \\
\hline pmol/L & $11.97-21.88$ & $>100.02$ & 36.69 & 18.02 & 24.59 & 21.50 \\
\hline ng/dL & $0.93-1.70$ & $>7.77$ & 2.85 & 1.40 & 1.91 & 1.67 \\
\hline \multicolumn{7}{|l|}{ T3 } \\
\hline $\mathrm{nmol} / \mathrm{L}$ & $0.93-2.61$ & $>10.00$ & 4.73 & 2.81 & & \\
\hline $\mathrm{ng} / \mathrm{dL}$ & $60.7-170$ & $>651$ & 308.0 & 182.6 & & \\
\hline $\mathrm{FSH}(\mathrm{mIU} / \mathrm{mL})$ & $1.5-12.4$ & & & 4 & & \\
\hline $\mathrm{LH}(\mathrm{mlU} / \mathrm{mL})$ & $1.70-8.60$ & & & 7.4 & & \\
\hline \multicolumn{7}{|l|}{ Total testosterone } \\
\hline $\mathrm{nmol} / \mathrm{L}$ & 8.63-29.99 & $>52.01$ & & & & \\
\hline $\mathrm{ng} / \mathrm{mL}$ & $2.49-8.36$ & $>15$ & & & & \\
\hline \multicolumn{7}{|l|}{ Estradiol } \\
\hline $\mathrm{pmol} / \mathrm{L}$ & $94.72-222.12$ & 323.45 & & 189.44 & & \\
\hline $\mathrm{pg} / \mathrm{mL}$ & $25.8-60.5$ & 88.1 & & 51.6 & & \\
\hline Anti-TSH receptor antibody (IU/L) & $0-1.1 .75$ & $>40$ & & & & \\
\hline Anti-TPO (IU/mL) & $0.00-34$ & $>600$ & & & & \\
\hline SHBG (nmol/L) & $16.5-55.9$ & & & 83.5 & & \\
\hline
\end{tabular}


to the development of gynecomastia can be the results of an increase in the production of estrogen, a decrease in androgen, alteration of serum estrogen-androgen ratio or androgen receptor defect (5). Pathological conditions associated with an increased level of estrogen to androgen ratio can range from tumor causes, including tumors of male reproductive system and adrenal tumors, to nontumor associated causes such as familial gynecomastia, polymorphism of cytochrome P450 family 19, obesity, thyroid disease, liver disease, end-stage renal disease and medications $(3,4)$.

Although the association of gynecomastia and hyperthyroidism was described for more than a century (6), the exact causes are still unclear. Previous studies showed that the manifestation of gynecomastia varied significantly among affected patients and had no correlation to the severity of hyperthyroid (1). It is proposed that the development of gynecomastia in hyperthyroid is involved with the balance between free androgen and estrogen, which is affected by elevated serum levels of sex hormonebinding globulin (SHBG). Thyroid hormones, particularly T4, influence plasma SHBG level by increasing hepatic production of SHBG (7). SHBG has a greater affinity in binding to androgen than estrogen, causing a decrease in the availability of free-androgen level. The decrease in free androgen induces negative feedback to the pituitary by increasing luteinizing hormone (LH). An increase in LH subsequently causes stimulation of Leydig cells to increase the production of androgen as well as estradiol (E2). LH not only enhances androgen and estrogen levels but also increases aromatase activity. Unfortunately, the increased level of E2 as a result of Leydig cell stimulation, along with increased peripheral tissue aromatization of androgen, contributes to the elevation of free-estrogen level in the patient. The pathophysiology described above explains the observed upregulation of both serum estradiol and total testosterone level in this patient. The level of SHBG in this patient was slightly increased after the start of treatment for 3 weeks, therefore, may not be as high as expected as in cases of hyperthyroidism, but the ratio of sex hormone at the start of treatment fits with the proposed mechanism of gynecomastia in hyperthyroid. These findings are consistent with previously reported cases in the past that higher levels of E2, unbound E2, E2/ testosterone ratio and LH were observed (8). Hyperthyroid patients with gynecomastia were found to have higher level of unbound E2, compared to those expressed in women (9). Interestingly, not all hyperthyroid patients with high levels of E2 develop symptoms of gynecomastia, thus additional factors such as duration of exposure to high levels of estrogen, target tissue responsiveness to hormone and increased aromatase activity may also contribute to the manifestation of this condition $(2,8)$.

The occurrence of gynecomastia in hyperthyroid can be as high as $40 \%$ as reported in the literature and this number is enough to warrant hyperthyroidism as a common differential diagnosis for the cause of pathologic bilateral gynecomastia. In contrast, the number of reported cases of unilateral gynecomastia in association with hyperthyroidism has been far fewer when compared to causes from breast cancer. The etiologies and mechanisms involved in the development of unilateral gynecomastia in hyperthyroidism remain to be elucidated. However, the manifestation of single breast tissue development seems to support the proposed mechanism involving target tissue responsiveness and an increase in aromatase activity. Specifically, unilateral breast tissue development in the hyperthyroid patient may result from different levels of target tissue responsiveness to increase estrogen between the left and right breast, causing one breast to enlarge more rapidly than the other breast (8). Furthermore, the local tissue of the left and right breasts may have different distribution of fat, which contributes to varying levels of aromatase activity. The breast with a greater composition of fat might have higher growth and eventually manifest as unilateral gynecomastia. The development of unilateral gynecomastia poses a challenge in diagnosis, since the growth of one breast is commonly associated with malignancy and rarely presented as a symptom of hyperthyroidism. Interestingly, this case developed unilateral gynecomastia and had symptoms of hyperthyroidism for only 1 month, but he had already developed enlarged thyroid and gynecomastia.

Regarding the treatment of gynecomastia in GD, the physician can choose either anti-thyroid medication or RAI to control thyroid hormone level similar to the standard indication of hyperthyroid treatment (10). In this case, we choose anti-thyroid medication first then followed by RAI for definite treatment since he developed severe symptoms of hyperthyroidism at a relatively young age. To the best of our knowledge, there is no definite guideline regarding the management of gynecomastia in hyperthyroidism. The treatment for this patient was based on few reported cases of gynecomastia in hyperthyroidism and on the clinical expertise in treating Graves' disease in Thai patients. According to the case reports, gynecomastia resolved once the patients were euthyroid. Therefore, our treatment goal for hyperthyroid-related gynecomastia was to achieve euthyroidism. After 3 months of treatment, symptoms of gynecomastia were completely resolved without 
complications of permanent breast fibrosis, and the patient remained in remission without relapse for 2 years. In conclusion, this case highlights the rare presentation of unilateral gynecomastia in hyperthyroidism from GD and the importance of precise diagnosis for prompt treatment of this reversible condition.

\section{Declaration of interest}

The authors declare that there is no conflict of interest that could be perceived as prejudicing the impartiality of the research reported.

\section{Funding}

This work did not receive any specific grant from any funding agency in the public, commercial or not-for-profit sector.

\section{Patient consent}

Written informed consent was obtained from the patient for publication of the submitted article.

\section{Author contribution statement}

T Sakulterdkiat wrote the initial draft of the article, and K Romphothong and all authors contributed to the editing process and oversight of the case report. Y Thewjitcharoen was involved in the direct care of the patient, investigation and follow-up of the patient.

\section{Acknowledgements}

Parts of this manuscript had previously been presented as a poster in AOTA 2019, the 13th Congress of Asia and Oceania Thyroid Association, Sydney, Australia.

\section{References}

1 Ashkar FS, Smoak WM, Gilson AJ \& Miller R. Gynecomastia and mastoplasia in Graves' disease. Metabolism: Clinical and Experimental 197019 946-951. (https://doi.org/10.1016/0026-0495(70)90041-7)

2 Braunstein GD. Clinical practice. Gynecomastia. New England Journal of Medicine 2007357 1229-1237. (https://doi.org/10.1056/ NEJMcp070677)

3 Dickson G. Gynecomastia. American Family Physician 201285 716-722. (https://pubmed.ncbi.nlm.nih.gov/22534349/)

4 Swerdloff RS \& Ng CM. Gynecomastia: etiology, diagnosis, and treatment. In Endotext [Internet]. Eds KR Feingold, B Anawalt, A Boyce, G Chrousos, WW de Herder, K Dhatariya, K Dungan, A Grossman, JM Hershman, J Hofland, et al. South Dartmouth (MA): MDText.com, Inc., 2019. (https://pubmed.ncbi.nlm.nih.gov/25905330/)

5 Carlson HE. Approach to the patient with gynecomastia. Journal of Clinical Endocrinology and Metabolism 201196 15-21. (https://doi. org/10.1210/jcem.96.9.zeg15a)

6 Sanyal T, Dutta D, Shivprasad K, Ghosh S, Mukhopadhyay S $\&$ Chowdhury S. Gynaecomastia as the initial presentation of thyrotoxicosis. Indian Journal of Endocrinology and Metabolism 2012 16 (Supplement 2) S352-S353. (https://doi.org/10.4103/22308210.104089)

7 David MS \& Geoffrey LH. Thyroid hormones act indirectly to increase sex hormone-binding globulin production by liver via hepatocyte nuclear factor-4a. Journal of Molecular Endocrinology 200943 19-27.

8 Chopra IJ \& Tulchinsky DAN. Status of estrogen-androgen balance in hyperthyroid men with Graves' disease. Journal of Clinical Endocrinology and Metabolism 197438 269-277. (https://doi. org/10.1210/jcem-38-2-269)

9 Chopra IJ, Abraham GE, Chopra U, Solomon DH \& Odell WD. Alterations in circulating estradiol-17 $\beta$ in male patients with Graves's disease. New England Journal of Medicine 1972286 124-129. (https:// doi.org/10.1056/NEJM197201202860303)

10 Calcaterra V, Clerici E, Ceolin V, Regalbuto C \& Larizza D. Gynecomastia after euthyroidism restoration in a patient with type 1 diabetes and Graves' disease. Clinical Case Reports 20186 1481-1484. (https://doi.org/10.1002/ccr3.1565)

Received in final form 24 May 202

Accepted 14 October 2021 